\title{
Solvability of Some Perturbed Generalized Variational Inequalities in Reflexive Banach Spaces
}

\author{
Xue-ping Luo \\ College of Computer Science and Technology, Southwest University for Nationalities, Chengdu 610041, China \\ Correspondence should be addressed to Xue-ping Luo; xuepingluo2014@outlook.com
}

Received 15 March 2018; Accepted 23 April 2018; Published 3 June 2018

Academic Editor: Adrian Petrusel

Copyright (c) 2018 Xue-ping Luo. This is an open access article distributed under the Creative Commons Attribution License, which permits unrestricted use, distribution, and reproduction in any medium, provided the original work is properly cited.

This paper aims to discuss the solvability of some perturbed generalized variational inequalities with both the mapping and the constraint set perturbed simultaneously in reflexive Banach spaces, under some coercivity conditions. In particular, a new result that the set is directional perturbed is presented. The main results generalize and extend some known results in this area.

\section{Introduction}

Throughout this paper, let us denote $X$ to be a reflexive Banach space with the dual space $X^{*}$, let $\|x\|$ be the norm of $x \in X$, and let $\langle\phi, x\rangle$ be the dual pair between $\phi \in X^{*}$ and $x \in X$. Let $K \subset X$ be a nonempty, closed, and convex subset and let $F: K \rightarrow 2^{X^{*}}$ be a set-valued mapping with nonempty values. We consider the following generalized variational inequality problem: to find $x \in K$ and $x^{*} \in F(x)$ such that

$$
\left\langle x^{*}, y-x\right\rangle \geq 0, \quad \forall y \in K,
$$

which has been extensively studied in the literature [1-9]. Here in this paper, we use $\operatorname{GVIP}(F, K)$ and $S(F, K)$ to denote problem (1) and its solution set, respectively.

If $F$ is single-valued, then (1) reduces to the following classical variational inequality $\operatorname{VIP}(F, K)[10,11]$ : to find $x \in$ $K$ such that

$$
\langle F(x), y-x\rangle \geq 0, \quad \forall y \in K .
$$

The variational inequalities have been widely studied by many authors in recent years; see [12-18]. One of the most interesting subjects in the theoretical aspect is the research of properties for variational inequalities under data perturbation [19-27]. Most of the research in the literature considered variational inequality where space is finite dimensional and the mapping $F$ is single-valued and continuous, and the mail tool for analyzing is the theory of topological degree. In particular, Corollary 5.5.12 in [11] applied topological degree as a theoretical tool to prove that if $X=\mathbb{R}^{n}$ and $F$ is singlevalued, then a coercivity condition implies that a perturbed variational inequality $\operatorname{VIP}(F+q, K)$ has a solution.

Assuming that the barrier cone of $K$ has nonempty interior, [28] showed a comprehensive study of the stability of the solution set of $\operatorname{GVIP}(F, K)$, where $F$ is maximal monotone. Later, He [4] discussed the stability analysis of $\operatorname{GVIP}(F, K)$ with either the mapping or the constraint set perturbed when $F$ is pseudomonotone, which is weaker than (maximal) monotone. In [3], Fan and Zhong further extended the main results of [4] to the case where the perturbation was imposed on the mapping and the constraint set simultaneously. Zhong and Huang [29] extended the results of $[3,4]$ to the mixed variational inequalities with $f$-pseudomonotone mappings. All the research mentioned above on the stability results considered variational inequalities when the mapping $F$ is set-valued and the theory of topological degree is not used in infinite dimensional spaces. Recently, $\mathrm{Li}$ and $\mathrm{He}$ [6] extended the aforementioned result of [11] by allowing $F$ to be a set-valued mapping with a weaker coercivity condition and presented the solvability of perturbed generalized variational inequalities in $\mathbb{R}^{n}$. Very recently, Li and Sun [30] generalized the main result of [6] from $\mathbb{R}^{n}$ to an infinite dimensional space and gave a stronger conclusion than Theorem 1.1 of [6].

Inspired and motivated by the works of $[6,30]$, in this paper, we establish the solvability of some perturbed generalized variational inequalities when both the mapping 
$F$ and the constraint set $K$ are perturbed simultaneously in reflexive Banach spaces. Our work improves and extends the main results of $[6,30]$ which considered only the mapping is perturbed. It is worth mentioning that Theorem 9 of this paper in which both the mapping and the constraint set are perturbed along some certain directions is new even if the mapping $F$ is single-valued.

The rest of this paper is organized as follows. In Section 2, we recall some notations and present some basic results. In Section 3, we consider the solvability of two different kinds of perturbed generalized variational inequalities with both the mapping $F$ and the constraint set $K$ perturbed simultaneously in reflexive Banach spaces. Finally, we conclude this paper in Section 4.

\section{Notations and Preliminaries}

Let $X, X^{*}$, and $K$ be as those in Section 1 . The symbols " $\rightarrow$ " and " $\rightarrow$ " are used to denote the strong and weak convergence, respectively. Let

$$
\operatorname{barr}(K):=\left\{x^{*} \in X^{*}: \sup _{x \in K}\left\langle x^{*}, x\right\rangle<\infty\right\}
$$

denoting the barrier cone of $K$. The recession cone of $K$ is the closed convex cone defined by

$$
K_{\infty}:=\left\{d \in X: \exists t_{n} \downarrow 0, \exists x_{n} \in K, t_{n} x_{n} \rightarrow d\right\} .
$$

It is known that, given $x_{0} \in K$,

$$
K_{\infty}=\left\{d \in X: x_{0}+\lambda d \in K, \forall \lambda>0\right\} .
$$

For a nonempty set $D$ in $X, D^{-}:=\left\{x^{*} \in X^{*}:\left\langle x^{*}, x\right\rangle \leq\right.$ $0, \forall x \in D\}$ and $\operatorname{int}(D)$ denotes the interior of $D$. By Proposition 3.10 in [31], we have

$$
\operatorname{barr}(K)^{-}=K_{\infty} .
$$

For a positive integer $m \in \mathbb{N}$, we denote $K_{m}:=\{x \in K$ : $\|x\| \leq m\}, \mathbb{B}(0, m):=\{x \in X:\|x\|<m\}$ and $\overline{\mathbb{B}}(0, m):=\{x \in$ $X:\|x\| \leq m\}$.

Definition 1. Let $F: K \rightarrow 2^{X^{*}}$ be a set-valued mapping with nonempty values. $F$ is said to be

(i) monotone on $K$ if for each pair of points $x, y \in K$ and for all $x^{*} \in F(x)$ and $y^{*} \in F(y)$,

$$
\left\langle y^{*}-x^{*}, y-x\right\rangle \geq 0
$$

(ii) quasimonotone on $K$ if for each pair of points $x, y \in$ $K$ and for all $x^{*} \in F(x)$ and $y^{*} \in F(y)$,

$$
\begin{aligned}
& \left\langle x^{*}, y-x\right\rangle>0 \Longrightarrow \\
& \left\langle y^{*}, y-x\right\rangle \geq 0 ;
\end{aligned}
$$

(iii) stably quasimonotone on $K$ with respect to a set $U \subset$ $X^{*}$ if $F$ and $F(\cdot)-u$ are quasimonotone on $K$ for every $u \in U$;

(iv) upper semicontinuous at $x_{0} \in K$ if for any neighborhood $\mathcal{N}\left(F\left(x_{0}\right)\right)$ of $F\left(x_{0}\right)$, there exists a neighborhood $\mathscr{N}\left(x_{0}\right)$ of $x_{0}$ such that

$$
F(x) \subset \mathcal{N}\left(F\left(x_{0}\right)\right), \quad \forall x \in \mathcal{N}\left(x_{0}\right) \cap K ;
$$

(v) upper hemicontinuous on $K$ if the restriction of $F$ to every line segment of $K$ is upper semicontinuous with respect to the weak topology in $X^{*}$.

Some preliminary results are quoted below.

Lemma 2 (see [4]). Let $K$ be a nonempty, closed, and convex subset in $X$ with $\operatorname{int}($ barr $K) \neq \emptyset$; then there does not exist $\left\{x_{n}\right\} \subset K$ with each $\left\|x_{n}\right\| \rightarrow \infty$ such that $x_{n} /\left\|x_{n}\right\| \rightarrow 0$. If additionally $K$ is a cone, then there does not exist $\left\{d_{n}\right\} \subset K$ with each $\left\|d_{n}\right\|=1$ such that $d_{n} \rightarrow 0$.

Lemma 3 (see [32]). If $K$ is a nonempty and convex subset of a Hausdorff topological vector space $E$ and $G: K \rightarrow 2^{E}$ is a set-valued mapping from $K$ into $E$ satisfying the following properties:

(i) $G$ is a KKM mapping for every finite subset $A$ of $K$, $\operatorname{co}(A) \subset \bigcup_{x \in A} G(x)$,

(ii) $G(x)$ is closed in $E$ for every $x \in K$,

(iii) $G\left(x_{0}\right)$ is compact in $E$ for some $x_{0} \in K$,

then $\bigcap_{x \in K} G(x) \neq \emptyset$.

Next we discuss the variational inequality property.

Definition 4. $F$ is said to have variational inequality property on $K$ if for every nonempty, bounded, closed, and convex $D$ of $K, \operatorname{GVIP}(F, D)$ has a solution.

Proposition 5. The following classes of mappings have the variational inequality property:

(i) Every weak-\|·\| upper semicontinuous set-valued mapping with nonempty compact convex values.

(ii) Every upper hemicontinuous and quasimonotone setvalued mapping with nonempty $w^{*}$-compact convex values.

(iii) If $F: K \rightarrow 2^{X^{*}}$ is a weak-\|.\| upper semicontinuous setvalued mapping with nonempty compact convex values and $q: K \rightarrow X^{*}$ is a strongly continuous mapping, then $F+q$ has the variational inequality property.

(iv) If $F: K \rightarrow 2^{X^{*}}$ is an upper hemicontinuous and stably quasimonotone set-valued mapping with nonempty $w^{*}$-compact convex values, then for any $\zeta \in X^{*}, F-\zeta$ has the variational inequality property.

Proof. (i) is well known in the literature and (ii) is verified in [7]. Let us now prove (iii). 
Let $D$ be a bounded, closed, and convex subset of $K$. Let $G: D \rightarrow 2^{D}$ be a set-valued mapping defined by

$$
\begin{aligned}
G(y):=\left\{x \in D: \sup _{\xi \in F(x)}\langle\xi+q(x), y-x\rangle \geq 0\right\}, & \\
& \forall y \in D .
\end{aligned}
$$

We first prove that $G(y)$ is a weakly closed subset of $D$. Indeed, let $x_{n} \in G(y)$ with $x_{n} \rightarrow x_{0}$. This means that, for some $\xi_{n} \in$ $F\left(x_{n}\right)$,

$$
\left\langle\xi_{n}+q\left(x_{n}\right), y-x_{n}\right\rangle \geq 0, \quad \forall y \in D .
$$

Since $F$ is weak- $\|\cdot\|$ upper semicontinuous with nonempty compact convex values, $\left\{\xi_{n}\right\}$ is compact. Without loss of generality, we assume $\lim _{n \rightarrow \infty} \xi_{n}=\xi$ for some $\xi \in F\left(x_{0}\right)$. Thus, for any $y \in D$, from $q$ is strongly continuous and (11), we have

$$
\sup _{\xi \in F\left(x_{0}\right)}\left\langle\xi+q\left(x_{0}\right), y-x_{0}\right\rangle \geq 0,
$$

which shows that $x_{0} \in G(y)$ and so $G(y)$ is sequentially weakly closed; being in addition a bounded subset of a Banach space, by virtue of the Eberlein-Šmulian theorem, $G(y)$ is automatically weakly closed. Since $X$ is reflexive Banach space, $G(y)$ is weakly compact in $D$ for any $y \in D$.

Next we prove that $G$ is a KKM map from $D$ to $D$. If it not true, then there exist $\lambda_{1}, \ldots, \lambda_{n} \in[0,1]$ with $\sum_{i=1}^{n} \lambda_{i}=1$, $\left\{y_{1}, \ldots, y_{n}\right\} \subset D$ and $\bar{y}=\sum_{i=1}^{n} \lambda_{i} y_{i}$ such that $\bar{y} \notin \bigcup_{i=1}^{n} G\left(y_{i}\right)$. It follows that

$$
\begin{aligned}
0 & =\sup _{\xi \in F(\bar{y})}\left\langle\xi, \sum_{i=1}^{n} \lambda_{i} y_{i}-\bar{y}\right\rangle+\left\langle q\left(x_{0}\right), \sum_{i=1}^{n} \lambda_{i} y_{i}-\bar{y}\right\rangle \\
& =\sum_{i=1}^{n} \lambda_{i}\left(\sup _{\xi \in F(\bar{y})}\left\langle\xi, y_{i}-\bar{y}\right\rangle+\left\langle q\left(x_{0}\right), y_{i}-\bar{y}\right\rangle\right)<0 .
\end{aligned}
$$

This contradiction shows that $G$ is a KKM map. By Lemma 3, there exists $\bar{x} \in \bigcap_{y \in D} G(y)$ and then $\bar{x}$ solves $\operatorname{GVIP}(F+q, D)$. Thus, $F+q$ has the variational inequality property. (ii).

Finally, (iv) can be proved similarly as Proposition 5 of

\section{Solvability of Some Perturbed Generalized Variational Inequalities}

This section is devoted to the main results in this paper, which include the solvability of perturbed generalized variational inequalities under two classes of perturbations allowed for the mapping $F$ and the set $K$ as follows:

(i) The mapping $F$ is perturbed by a nonlinear mapping and the set $K$ is perturbed by a closed unit ball.

(ii) The mapping $F$ is perturbed along a direction and the set $K$ is perturbed along another direction.

They are discussed in Sections 3.1 and 3.2, respectively.
3.1. Nonlinear Perturbed Mapping and Ball Perturbed Set. In this subsection, we denote $K^{\mu}$ to be the set $K+\mu \overline{\mathbb{B}}$ for any $\mu>0$ with $\overline{\mathbb{B}}$ being the closed unit ball in $X$ and $\mathbb{B}\left(0 ; \varepsilon,\left(K^{\mu}\right)_{m}\right)$ to be the set of strongly continuous and monotone mappings $q: K^{\mu} \rightarrow X^{*}$ satisfying $\|q(x)\|<\varepsilon$ for all $x \in\left(K^{\mu}\right)_{m}$.

Theorem 6. Let $K$ be a nonempty, closed, and convex subset in X. Suppose that, for any $\mu>0, F: K^{\mu} \rightarrow 2^{X^{*}}$ is a weak$\|\cdot\|$ upper semicontinuous mapping with nonempty compact convex values. If the following coercivity condition holds:

$$
\begin{aligned}
& \exists r>0, \forall x \in K \backslash K_{r}, \exists y \in K_{r} \\
& \text { such that } \sup _{x^{*} \in F(x)}\left\langle x^{*}, y-x\right\rangle<0,
\end{aligned}
$$

then for every $m>r$, there exist $\varepsilon>0$ and $\delta>0$ such that

$$
\begin{aligned}
& \emptyset \neq S(F+q, K+\theta \overline{\mathbb{B}}) \subset \overline{\mathbb{B}}(0, m), \\
& \forall q \in \mathbb{B}\left(0 ; \varepsilon,\left(K^{\delta}\right)_{m}\right), \forall \theta \in(0, \delta) .
\end{aligned}
$$

Proof. (1) Firstly, we need to prove that, for every $m>r$, there exist $\varepsilon>0$ and $\delta>0$, for any $q \in \mathbb{B}\left(0 ; \varepsilon,\left(K^{\delta}\right)_{m}\right)$ and $\theta \in(0, \delta)$ such that $S(F+q, K+\theta \overline{\mathbb{B}}) \neq \emptyset$. If not, then there exists $m>r$, for every $\varepsilon>0$ and every $\delta>0$, there exist $q_{\varepsilon} \in \mathbb{B}\left(0 ; \varepsilon,\left(K^{\delta}\right)_{m}\right)$ and $\theta_{\delta} \in(0, \delta)$ such that $F$ is weak$\|\cdot\|$ upper semicontinuous with nonempty compact convex values on $K^{\theta_{\delta}}$ and $S\left(F+q_{\varepsilon}, K+\theta_{\delta} \overline{\mathbb{B}}\right)=\emptyset$.

Denote $L_{m}:=\left\{x \in K^{\theta_{\delta}}:\|x\| \leq m\right\}$. By the fact that $F$ is weak- $\|\cdot\|$ upper semicontinuous with nonempty compact convex values and Proposition 5(iii), $F+q_{\varepsilon}$ has the variational inequality property on $K^{\theta_{\delta}}$. Since $L_{m}$ is bounded, closed, and convex, there exists $x_{\varepsilon}^{\delta} \in L_{m}$ such that

$$
\sup _{\xi \in F\left(x_{\varepsilon}^{\delta}\right)}\left\langle\xi+q_{\varepsilon}\left(x_{\varepsilon}^{\delta}\right), y-x_{\varepsilon}^{\delta}\right\rangle \geq 0, \quad \forall y \in L_{m} .
$$

(i) If for some $\varepsilon>0$ and some $\delta>0,\left\|x_{\varepsilon}^{\delta}\right\|<m$, then for any $y \in K+\theta_{\delta} \overline{\mathbb{B}}$, there exists $t \in(0,1)$ such that $z_{t}:=$ $x_{\varepsilon}^{\delta}+t\left(y-x_{\varepsilon}^{\delta}\right) \in L_{m}$, because of the convexity of $K+\theta_{\delta} \frac{\overline{\mathbb{B}}}{\text {. It }}$ follows from (16) that

$$
\begin{aligned}
0 & \leq \sup _{\xi \in F\left(x_{\varepsilon}^{\delta}\right)}\left\langle\xi+q_{\varepsilon}\left(x_{\varepsilon}^{\delta}\right), z_{t}-x_{\varepsilon}^{\delta}\right\rangle \\
& =t \sup _{\xi \in F\left(x_{\varepsilon}^{\delta}\right)}\left\langle\xi+q_{\varepsilon}\left(x_{\varepsilon}^{\delta}\right), y-x_{\varepsilon}^{\delta}\right\rangle .
\end{aligned}
$$

Since $y \in K+\theta_{\delta} \overline{\mathbb{B}}$ is arbitrary, $x_{\varepsilon}^{\delta}$ solves $\operatorname{GVIP}\left(F+q_{\varepsilon}, K+\theta_{\delta} \overline{\mathbb{B}}\right)$. Thus, $x_{\varepsilon}^{\delta} \in S\left(F+q_{\varepsilon}, K+\theta_{\delta} \overline{\mathbb{B}}\right) \neq \emptyset$.

(ii) If for each $\varepsilon>0$ and each $\delta>0,\left\|x_{\varepsilon}^{\delta}\right\|=m$. Without loss of generality, we may assume that $x_{\varepsilon}^{\delta} \rightarrow d$ as $\varepsilon \rightarrow 0^{+}$and $\delta \rightarrow 0^{+}$. Since $x_{\varepsilon}^{\delta} \in K+\theta_{\delta} \overline{\mathbb{B}}$, there exists $\widetilde{x_{\varepsilon}^{\delta}} \in K$ such that

$$
\lim _{\delta \rightarrow 0^{+}}\left\|x_{\varepsilon}^{\delta}-\widetilde{x_{\varepsilon}^{\delta}}\right\|=0
$$

This together with the weak convergence of $x_{\varepsilon}^{\delta}$ to $d$ yield that $\widetilde{x_{\varepsilon}^{\delta}} \rightarrow d$. Thus, we can obtain $d \in K$ because $K$ is 
closed and convex and hence weakly closed. Moreover, as $\left|\left\|x_{\varepsilon}^{\delta}\right\|-\left\|\widetilde{x_{\varepsilon}^{\delta}}\right\|\right| \leq\left\|x_{\varepsilon}^{\delta}-\widetilde{x_{\varepsilon}^{\delta}}\right\|$ and $m>r$, we have $\widetilde{x_{\varepsilon}^{\delta}} \notin K_{r}$ for small enough $\delta>0$. Thus, the coercivity condition (14) implies that, for small enough $\delta>0$, there exists $y_{\varepsilon}^{\delta} \in K_{r}$ such that $\sup _{\eta \in F\left(\widetilde{x_{\varepsilon}^{\delta}}\right)}\left\langle\eta, y_{\varepsilon}^{\delta}-\widetilde{x_{\varepsilon}^{\delta}}\right\rangle<0$. By $\left\|y_{\varepsilon}^{\delta}\right\| \leq r<m$, without loss of generality, $y_{\varepsilon}^{\delta} \rightarrow y_{0}$ as $\varepsilon \rightarrow 0^{+}$and $\delta \rightarrow 0^{+}$. As $F$ is weak- $\|\cdot\|$ upper semicontinuous and $\widetilde{x_{\varepsilon}^{\delta}} \rightarrow d$, we have $\sup _{\zeta \in F(d)}\left\langle\zeta, y_{0}-d\right\rangle \leq 0$.

Next we claim that, for $\varepsilon, \delta>0$ small enough, $\sup _{\xi \in F\left(x_{\varepsilon}^{\delta}\right)}\left\langle\xi, y_{\varepsilon}^{\delta}-x_{\varepsilon}^{\delta}\right\rangle<0$. Indeed, $x_{\varepsilon}^{\delta} \rightarrow d$ implies every weak neighborhood $U$ of $d$ such that $x_{\varepsilon}^{\delta} \in U$ with sufficiently small $\varepsilon>0$ and $\delta>0$. Moreover, as the set $\left\{\zeta \in X^{*}:\left\langle\zeta, y_{0}-d\right\rangle \leq 0\right\}$ is a neighborhood of $F(d)$ for the weakly* topology and $F$ is weak-\| \|\| upper semicontinuous, for any $\xi_{\varepsilon}^{\delta} \in F\left(x_{\varepsilon}^{\delta}\right)$, we have $\left\langle\xi_{\varepsilon}^{\delta}, y_{0}-d\right\rangle \leq 0$. Since $F$ has nonempty compact convex values, $\left\{\xi_{\varepsilon}^{\delta}\right\}$ is compact. Without of loss of generality, $\xi_{\varepsilon}^{\delta} \rightarrow \xi_{0}$ for some $\xi_{0} \in F(d)$. It follows that

$$
\lim _{\varepsilon \rightarrow 0^{+}, \delta \rightarrow 0^{+}}\left\langle\xi_{\varepsilon}^{\delta}, y_{\varepsilon}^{\delta}-x_{\varepsilon}^{\delta}\right\rangle=\left\langle\xi_{0}, y_{0}-d\right\rangle \leq 0,
$$

and so for $\varepsilon, \delta>0$ small enough, we have the claim.

Now, as $x_{\varepsilon}^{\delta} \in K^{\theta_{\delta}} \subset K^{\delta}$ and $\left\|x_{\varepsilon}^{\delta}\right\|=m$, we have $x_{\varepsilon}^{\delta} \epsilon$ $\left(K^{\delta}\right)_{m}$. Therefore, it follows from $\sup _{x \in\left(K^{\delta}\right)_{m}}\left\|q_{\varepsilon}(x)\right\|<\varepsilon$ that

$$
\lim _{\varepsilon \rightarrow 0^{+}, \delta \rightarrow 0^{+}}\left\langle q_{\varepsilon}\left(x_{\varepsilon}^{\delta}\right), y_{\varepsilon}^{\delta}-x_{\varepsilon}^{\delta}\right\rangle=0 .
$$

Thus, there exist $\varepsilon_{1}>0$ and $\delta_{1}>0$ such that

$$
\begin{aligned}
\sup _{\xi \in F\left(x_{\varepsilon}^{\delta}\right)}\left\langle\xi+q_{\varepsilon}\left(x_{\varepsilon}^{\delta}\right), y_{\varepsilon}^{\delta}-x_{\varepsilon}^{\delta}\right\rangle & <0, \\
\forall \varepsilon & \in\left(0, \varepsilon_{1}\right), \forall \delta \in\left(0, \delta_{1}\right) .
\end{aligned}
$$

Since $\left\|y_{\varepsilon}^{\delta}\right\|<m$, for any $y \in K+\theta_{\delta} \overline{\mathbb{B}}$, there exists $\lambda \in(0,1)$ such that $z_{\lambda}^{\delta}:=y_{\varepsilon}^{\delta}+\lambda\left(y-y_{\varepsilon}^{\delta}\right)$ and $\left\|z_{\lambda}^{\delta}\right\| \leq m$. Now we claim that, for $\delta>0$ small enough, $z_{\lambda}^{\delta} \in K+\theta_{\delta} \bar{B}$. Indeed, in view of $y_{\varepsilon}^{\delta} \in K$, there exist $y_{1} \in K+\theta_{\delta} \overline{\mathbb{B}}$ and $b \in \theta_{\delta} \overline{\mathbb{B}}$ such that $y_{\varepsilon}^{\delta}=y_{1}-b$. Thus, $z_{\lambda}^{\delta}=y_{1}+\lambda\left(y-y_{1}\right)-(1-\lambda) b$ and $z_{\lambda}^{\delta}$ converges to $y_{1}+\lambda\left(y-y_{1}\right)$ in the norm topology as $\delta \rightarrow 0+$. Since $K+\theta_{\delta} \overline{\mathbb{B}}$ is convex, $y_{1}+\lambda\left(y-y_{1}\right) \in K+\theta_{\delta} \overline{\mathbb{B}}$. It follows that there exists $\gamma>0$ such that

$$
z_{\lambda}^{\delta} \in K+\theta_{\delta} \overline{\mathbb{B}}, \quad \forall \delta \in(0, \gamma) .
$$

Therefore, $z_{\lambda}^{\delta} \in L_{m}$ for sufficiently small $\delta>0$.

By (16) and (21), for any $\varepsilon \in\left(0, \varepsilon_{1}\right)$ and any $\delta \in$ $\left(0, \min \left\{\delta_{1}, \gamma\right\}\right)$, we have

$$
\begin{aligned}
0 \leq & \sup _{\xi \in F\left(x_{\varepsilon}^{\delta}\right)}\left\langle\xi+q_{\varepsilon}\left(x_{\varepsilon}^{\delta}\right), z_{\lambda}^{\delta}-x_{\varepsilon}^{\delta}\right\rangle \\
= & \lambda \sup _{\xi \in F\left(x_{\varepsilon}^{\delta}\right)}\left\langle\xi+q_{\varepsilon}\left(x_{\varepsilon}^{\delta}\right), y-x_{\varepsilon}^{\delta}\right\rangle \\
& +(1-\lambda) \sup _{\xi \in F\left(x_{\varepsilon}^{\delta}\right)}\left\langle\xi+q_{\varepsilon}\left(x_{\varepsilon}^{\delta}\right), y_{\varepsilon}^{\delta}-x_{\varepsilon}^{\delta}\right\rangle \\
< & \lambda \sup _{\xi \in F\left(x_{\varepsilon}^{\delta}\right)}\left\langle\xi+q_{\varepsilon}\left(x_{\varepsilon}^{\delta}\right), y-x_{\varepsilon}^{\delta}\right\rangle .
\end{aligned}
$$

Since $y \in K+\theta_{\delta} \overline{\mathbb{B}}$ is arbitrary, $x_{\varepsilon}^{\delta}$ solves $\operatorname{GVIP}\left(F+q_{\varepsilon}, K+\theta_{\delta} \overline{\mathbb{B}}\right)$. It shows that $x_{\varepsilon}^{\delta} \in S\left(F+q_{\varepsilon}, K+\theta_{\delta} \overline{\mathbb{B}}\right) \neq \emptyset$ for sufficiently small $\varepsilon>0$ and $\delta>0$.

In either case, we obtain a contradiction to $S\left(F+q_{\varepsilon}, K+\right.$ $\left.\theta_{\delta} \overline{\mathbb{B}}\right)=\emptyset$.

(2) Now we need to prove that, for every $m>r$, there exist $\varepsilon>0$ and $\delta>0$, for any $q \in \mathbb{B}\left(0 ; \varepsilon,\left(K^{\delta}\right)_{m}\right)$ and $\theta \in(0, \delta)$ such that $S(F+q, K+\theta \overline{\mathbb{B}}) \subset \overline{\mathbb{B}}(0, m)$. By the first part (1), $S(F+q, K+\theta \overline{\mathbb{B}}) \neq \emptyset$. Suppose that there exists $m_{0}>r$, and for any $\widehat{\varepsilon}>0$ and $\widehat{\delta}>0$, there exist $\widetilde{q_{\widehat{\varepsilon}}} \in \mathbb{B}\left(0 ; \widehat{\mathcal{\varepsilon}},\left(K^{\widehat{\delta}}\right)_{m_{0}}\right)$ and $\widetilde{\theta_{\widehat{\delta}}} \in(0, \widehat{\delta})$ such that $x_{\widehat{\varepsilon}}^{\widehat{\delta}} \in S\left(F+\widetilde{q_{\widehat{\varepsilon}}}, K+\widetilde{\theta_{\widehat{\delta}}} \overline{\mathbb{B}}\right)$; however, $x_{\widetilde{\varepsilon}}^{\widehat{\delta}} \notin$ $\overline{\mathbb{B}}\left(0, m_{0}\right)$.

Since $x_{\widehat{\varepsilon}}^{\widehat{\delta}} \in K+\widetilde{\theta_{\widehat{\delta}}} \overline{\mathbb{B}}$ and $\left\|x_{\widehat{\varepsilon}}^{\widehat{\delta}}\right\|>m_{0}>r$, applying the same method of proof as (1), there exist $\widetilde{x_{\widetilde{\varepsilon}}^{\hat{\delta}}} \in K$ and $\left\|x_{\widehat{\widetilde{\varepsilon}}}^{\widehat{\delta}}\right\|>r$ for small enough $\widehat{\delta}>0$. It follows from coercivity condition (14) that there exists $y_{\widehat{\varepsilon}}^{\widehat{\delta}} \in K_{r}$ such that $\sup _{\zeta \in F\left(\widetilde{x_{\tilde{\varepsilon}}^{\delta}}\right)}\left\langle\zeta, y_{\widehat{\varepsilon}}^{\widehat{\delta}}-\widetilde{x_{\widehat{\varepsilon}}^{\widehat{\delta}}}\right\rangle<0$. Similarly, we can get

$$
\sup _{\xi \in F\left(x_{\widehat{\varepsilon}}^{\widehat{\delta}}\right)}\left\langle\xi, y_{\widehat{\varepsilon}}^{\widehat{\delta}}-x_{\widehat{\varepsilon}}^{\widehat{\delta}}\right\rangle<0 .
$$
have

By $y_{\widehat{\varepsilon}}^{\widehat{\delta}} \in K \subset K+\widetilde{\theta_{\widehat{\delta}}} \bar{B}$ and $x_{\widehat{\varepsilon}}^{\widehat{\delta}} \in S\left(F+\widetilde{q_{\widehat{\varepsilon}}}, K+\widetilde{\theta_{\widehat{\delta}}} \overline{\mathbb{B}}\right)$, we

$$
\begin{aligned}
0 & \leq \sup _{\xi^{*} \in F\left(x_{\tilde{\varepsilon}}^{\widehat{\delta}}\right)}\left\langle\xi^{*}+\widetilde{q_{\widehat{\varepsilon}}}\left(x_{\widehat{\varepsilon}}^{\widehat{\delta}}\right), y_{\widehat{\varepsilon}}^{\widehat{\delta}}-x_{\widehat{\varepsilon}}^{\widehat{\delta}}\right\rangle \\
& =\sup _{\xi^{*} \in F\left(x_{\tilde{\varepsilon}}^{\widehat{\delta}}\right)}\left\langle\xi^{*}, y_{\widehat{\varepsilon}}^{\widehat{\delta}}-x_{\widehat{\varepsilon}}^{\widehat{\delta}}\right\rangle+\left\langle\widetilde{q_{\widehat{\varepsilon}}}\left(x_{\widehat{\varepsilon}}^{\widehat{\delta}}\right), y_{\widehat{\varepsilon}}^{\widehat{\delta}}-x_{\widehat{\varepsilon}}^{\widehat{\delta}}\right\rangle \\
& <\left\langle\widetilde{q_{\widehat{\varepsilon}}}\left(x_{\widehat{\varepsilon}}^{\widehat{\delta}}\right), y_{\widehat{\varepsilon}}^{\widehat{\delta}}-x_{\widehat{\varepsilon}}^{\widehat{\delta}}\right\rangle .
\end{aligned}
$$

Moreover, $\left\|y_{\hat{\varepsilon}}^{\widehat{\delta}}\right\| \leq r<m_{0}$ implies that

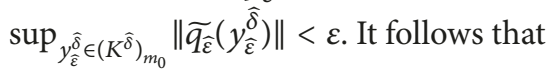

$$
\lim _{\widehat{\varepsilon} \rightarrow 0^{+}, \widehat{\delta} \rightarrow 0^{+}}\left\langle\widetilde{q_{\widehat{\varepsilon}}}\left(y_{\widehat{\varepsilon}}^{\widehat{\delta}}\right), y_{\widehat{\varepsilon}}^{\widehat{\delta}}-x_{\widehat{\varepsilon}}^{\widehat{\delta}}\right\rangle=0
$$

Thus, there exist small enough $\widehat{\varepsilon}>0$ and $\widehat{\delta}>0$ such that

$$
\left\langle\widetilde{q_{\widehat{\varepsilon}}}\left(y_{\widehat{\varepsilon}}^{\widehat{\delta}}\right)-\widetilde{q_{\widehat{\varepsilon}}}\left(x_{\widehat{\varepsilon}}^{\widehat{\delta}}\right), y_{\widehat{\varepsilon}}^{\widehat{\delta}}-x_{\widehat{\varepsilon}}^{\widehat{\delta}}\right\rangle<0 .
$$

We obtain a contradiction from the monotonicity of $\widetilde{q_{\bar{\varepsilon}}}$. This completes the proof.

Remark 7. Li and He [6] gave a similar result where only the mapping was perturbed in $\mathbb{R}^{n}$ (Theorem 3.1 of [6]). Theorem 6 generalizes the result of [6] from $\mathbb{R}^{n}$ to a reflexive Banach space, gives a stronger conclusion, and discusses the case that the mapping is perturbed by a nonlinear mapping and the constraint set is perturbed by a closed unit ball simultaneously. 
3.2. Directional Perturbed Mapping and Directional Perturbed Set. Let us turn to the case that the mapping $F$ is perturbed along a direction and the set $K$ is perturbed along another direction. Here in this section, we use $I$ to denote the set $K-$ $t K_{\infty} \backslash\{0\}$ for any $t>0$.

Lemma 8. If $K$ is a nonempty, closed, and convex subset in $X$, then $K_{\infty}=I_{\infty}$ and $\operatorname{barr}(K)=\operatorname{barr}(I)$.

Proof. Let $d \in I_{\infty}$. Given $x_{0} \in K$, in view of $K:=I+t K_{\infty} \backslash\{0\}$, there exist $y_{0} \in I$ and $\bar{d} \in K_{\infty} \backslash\{0\}$ such that $x_{0}=y_{0}+t \bar{d}$. It follows that, for all $\lambda>0$,

$$
\begin{aligned}
x_{0}+\lambda d & =\left(y_{0}+t \bar{d}\right)+\lambda d=\left(y_{0}+\lambda d\right)+t \bar{d} \\
& \in I+t K_{\infty} \backslash\{0\}=K,
\end{aligned}
$$

and so $d \in K_{\infty}$. This implies that $I_{\infty} \subset K_{\infty}$.

Next we prove the converse inclusion. Let $d_{1} \in K_{\infty}$. Given $x_{1} \in I$, in view of $I:=K-t K_{\infty} \backslash\{0\}$, there exist $y_{1} \in K$ and $\overline{d_{1}} \in K_{\infty} \backslash\{0\}$ such that $x_{1}=y_{1}-t \overline{d_{1}}$. It follows that, for all $\lambda_{1}>0$,

$$
\begin{aligned}
x_{1}+\lambda_{1} d_{1} & =\left(y_{1}-t \overline{d_{1}}\right)+\lambda_{1} d_{1}=\left(y_{1}+\lambda d_{1}\right)-t \overline{d_{1}} \\
& \in K-t K_{\infty} \backslash\{0\}=I,
\end{aligned}
$$

and so $d_{1} \in I_{\infty}$, implying that $K_{\infty} \subset I_{\infty}$. Therefore, $K_{\infty}=I_{\infty}$.

Finally, by (6), we have $\operatorname{barr}(K)=\operatorname{barr}(I)$.

Theorem 9. Let $K$ be a nonempty, closed, and convex subset in $X$. Suppose that $\operatorname{int}($ barr $K) \neq \emptyset$ and that $F: I \rightarrow 2^{X^{*}}$ is an upper hemicontinuous and stably quasimonotone setvalued mapping with nonempty $w^{*}$-compact convex values. If the following coercivity condition holds:

$$
\exists r>0, \forall x \in K \backslash K_{r}, \exists y \in K_{r}
$$

such that $\exists y^{*} \in F(y) \cap \operatorname{barr}(K),\left\langle y^{*}, y-x\right\rangle<0$,

then for any $q \in \operatorname{int}($ barr $K)$, there exists $k>r$ such that

$$
\begin{aligned}
\emptyset \neq S\left(F-\varepsilon q, K-t K_{\infty} \backslash\{0\}\right) \subset \overline{\mathbb{B}}\left(0, \frac{1}{\varepsilon}\right), \\
\forall \varepsilon \in\left(0, \frac{1}{k}\right), \forall t>0 .
\end{aligned}
$$

Proof. (1) Firstly, we need to prove that, for any $q \epsilon$ $\operatorname{int}($ barr $K)$, there exists $m>r$ such that, for every $\varepsilon \in$ $(0,1 / m), S\left(F-\varepsilon q, K-t K_{\infty} \backslash\{0\}\right) \neq \emptyset$. Suppose that there exists $q \in \operatorname{int}(\operatorname{barr} K)$, and for any $m>r$, there exists $\varepsilon_{m} \in(0,1 / m)$ such that $S\left(F-\varepsilon_{m} q, K-t K_{\infty} \backslash\{0\}\right)=\emptyset$.

Denote $E_{m}:=\left\{x \in I:\|x\| \leq 1 / \varepsilon_{m}\right\}$. By that $E_{m}$ is bounded, closed, and convex, $F$ is upper hemicontinuous and stably quasimonotone set-valued mapping with nonempty $w^{*}$-compact convex values, and Proposition 5(iv), $\operatorname{GVIP}(F-$ $\left.\varepsilon_{m} q, E_{m}\right)$, has a solution. For each $m \in \mathbb{N}$, there exists $x_{m} \in E_{m}$ such that

$$
\sup _{\xi \in F\left(x_{m}\right)}\left\langle\xi-\varepsilon_{m} q, y-x_{m}\right\rangle \geq 0, \quad \forall y \in E_{m} .
$$

(i) If for some $m>r,\left\|x_{m}\right\|<1 / \varepsilon_{m}$, then for any $y \in$ $K-t K_{\infty} \backslash\{0\}$, there exists $\lambda \in(0,1)$ such that $z_{\lambda}:=x_{m}+$ $\lambda\left(y-x_{m}\right) \in E_{m}$. This is because the convexity of $K$ and $K_{\infty}$ implies that $K-t K_{\infty} \backslash\{0\}$ is convex. It follows that

$$
\begin{aligned}
0 & \leq \sup _{\xi \in F\left(x_{m}\right)}\left\langle\xi-\varepsilon_{m} q, z_{\lambda}-x_{m}\right\rangle \\
& =t \sup _{\xi \in F\left(x_{m}\right)}\left\langle\xi-\varepsilon_{m} q, y-x_{m}\right\rangle .
\end{aligned}
$$

Since $y \in K-t K_{\infty} \backslash\{0\}$ is arbitrary, $x_{m}$ solves $\operatorname{GVIP}(F-$ $\left.\varepsilon_{m} q, K-t K_{\infty} \backslash\{0\}\right)$. Thus, $x_{m} \in S\left(F-\varepsilon_{m} q, K-t K_{\infty} \backslash\{0\}\right) \neq \emptyset$.

(ii) If for each $m>r,\left\|x_{m}\right\|=1 / \varepsilon_{m}>m$. Since $x_{m} \in$ $K-t K_{\infty} \backslash\{0\}$, there exist $\widetilde{x_{m}} \in K$ and $p_{m} \in K_{\infty} \backslash\{0\}$ such that $x_{m}=\widetilde{x_{m}}-t p_{m}$. We claim that $\widetilde{x_{m}} \notin K_{r}$. Indeed, if not, then $\left\|\widetilde{x_{m}}\right\| \leq r$. Thus,

$$
t\left\|p_{m}\right\| \leq\left\|x_{m}\right\|+\left\|\widetilde{x_{m}}\right\| \leq \frac{1}{\varepsilon_{m}}+r<\frac{2}{\varepsilon_{m}} .
$$

It follows that $t\left\|p_{m}\right\|<+\infty$ for each $m$. However, by $p_{m} \in$ $K_{\infty} \backslash\{0\}$, we have $\left\|p_{m}\right\|>0$. Letting $t \rightarrow+\infty$, this implies that $t\left\|p_{m}\right\|$ has no upper bound for each $m>r$. Thus, we obtain a contradiction and so the claim is proved. The coercivity condition (30) implies that there exists $y_{m} \in K_{r}$ such that there exists $y_{m}^{*} \in F\left(y_{m}\right) \cap \operatorname{barr}(K),\left\langle y_{m}^{*}, y_{m}-\widetilde{x_{m}}\right\rangle<0$. Thus, by (6), we have

$$
\begin{aligned}
\left\langle y_{m}^{*}, y_{m}-x_{m}\right\rangle & =\left\langle y_{m}^{*}, y_{m}-\widetilde{x_{m}}\right\rangle+\left\langle y_{m}^{*}, \widetilde{x_{m}}-x_{m}\right\rangle \\
& <t\left\langle y_{m}^{*}, p_{m}\right\rangle \leq 0,
\end{aligned}
$$

that is, for large enough $m \inf _{y_{m}^{*} \in F\left(y_{m}\right)}\left\langle y_{m}^{*}, y_{m}-x_{m}\right\rangle<0$ holds. It follows from the quasimonotonicity of $F$ that

$$
\sup _{\xi \in F\left(x_{m}\right)}\left\langle\xi, y_{m}-x_{m}\right\rangle \leq 0
$$

Without loss of generality, we may assume that $x_{m} /\left\|x_{m}\right\| \rightarrow d \in I_{\infty}$. By Lemma 8, we have $d \in K_{\infty}$. As $\operatorname{int}(\operatorname{barr} I)=\operatorname{int}(\operatorname{barr} K) \neq \emptyset$, Lemma 2 implies $d \neq 0$. From $q \in \operatorname{int}($ barr $K)$ and (6), we obtain $\langle q, d\rangle \leq 0$. We claim that $\langle q, d\rangle\langle 0$. In fact, if not, then $\langle q, d\rangle=0$. Since $q \in \operatorname{int}(\operatorname{barr} K)$, for any $x^{*} \in X^{*}$, there exists $\delta \in(0,1)$ such that $\delta q+(1-\delta) x^{*} \in$ barr $K$. It implies that $\left\langle\delta q+(1-\delta) x^{*}, d\right\rangle \leq 0$ and so $\left\langle x^{*}, d\right\rangle \leq 0$. Similarly, we can obtain $-\left\langle x^{*}, d\right\rangle \leq 0$. Thus, $\left\langle x^{*}, d\right\rangle=0$ which contradicts with $d \neq 0$. Therefore, $\langle q, d\rangle<0$.

Since $\left\|y_{m}\right\|<r<1 / \varepsilon_{m}$, then for any $y \in K-t K_{\infty} \backslash\{0\}$, there exists $\theta \in(0,1)$ such that $z_{\theta}:=y_{m}+\theta\left(y-y_{m}\right)$ and $\left\|z_{\theta}\right\| \leq 1 / \varepsilon_{m}$. Let us now claim that $z_{\theta} \in I$. Indeed, in view of $I:=K-t K_{\infty} \backslash\{0\}$ and $y_{m} \in K$, there exist $\widetilde{y_{m}} \in I$ and $p_{m} \in K_{\infty} \backslash\{0\}$ such that $y_{m}=\widetilde{y_{m}}+p_{m}$, and hence $z_{\theta}=$ $\widetilde{y_{m}}+\theta\left(y-\widetilde{y_{m}}\right)+(1-\theta) p_{m}$. Since $I$ is convex, $\widetilde{y_{m}}+\theta\left(y-\widetilde{y_{m}}\right) \in I$. From Lemma 8 , we know $p_{m} \in K_{\infty}=I_{\infty}$. By the definition of the recession cone and $1-\theta>0$, we get $z_{\theta} \in I$. Therefore, $z_{\theta} \in E_{m}$.

It follows from (32) and (36) that

$$
\begin{aligned}
0 & \leq \sup _{\xi \in F\left(x_{m}\right)}\left\langle\xi-\varepsilon_{m} q, z_{\theta}-x_{m}\right\rangle \\
& =\theta \sup _{\xi \in F\left(x_{m}\right)}\left\langle\xi-\varepsilon_{m} q, y-x_{m}\right\rangle
\end{aligned}
$$




$$
\begin{gathered}
+(1-\theta) \sup _{\xi \in F\left(x_{m}\right)}\left\langle\xi, y_{m}-x_{m}\right\rangle \\
+\varepsilon_{m}(1-\theta)\left(\left\langle q, x_{m}\right\rangle-\left\langle q, y_{m}\right\rangle\right) \\
\leq \theta \sup _{\xi \in F\left(x_{m}\right)}\left\langle\xi-\varepsilon_{m} q, y-x_{m}\right\rangle \\
+\varepsilon_{m}(1-\theta)\left(\left\langle q, x_{m}\right\rangle-\left\langle q, y_{m}\right\rangle\right) .
\end{gathered}
$$

Since $\varepsilon_{m}=1 /\left\|x_{m}\right\|, \varepsilon_{m}\left\langle q, x_{m}\right\rangle=\left\langle q, x_{m} /\left\|x_{m}\right\|\right\rangle \rightarrow\langle q, d\rangle\langle 0$. By sign-preserving theorem of limit, there exists $M>0$, when $m>M, \varepsilon_{m}\left\langle q, x_{m}\right\rangle<(1 / 2)\langle q, d\rangle<0$.

Also noticing that $\left\{y_{m}\right\} \subset K_{r},\left\{y_{m}\right\}$ is bounded, we have $\varepsilon_{m}\left\langle q, y_{m}\right\rangle \rightarrow 0$. It follows that, for large enough $m$,

$$
\sup _{\xi \in F\left(x_{m}\right)}\left\langle\xi-\varepsilon_{m} q, y-x_{m}\right\rangle \geq 0
$$

As $y \in K-t K_{\infty} \backslash\{0\}$ is arbitrary, $x_{m}$ solves $\operatorname{GVIP}\left(F-\varepsilon_{m} q, K-\right.$ $\left.t K_{\infty} \backslash\{0\}\right)$.

In either case, we obtain a contradiction to $S\left(F-\varepsilon_{m} q, K-\right.$ $\left.t K_{\infty} \backslash\{0\}\right)=\emptyset$.

(2) Now we need to prove that, for any $q \in \operatorname{int}(\operatorname{barr} K)$, there exists $k \geq m>r$ such that, for every $\varepsilon \in(0,1 / k), S(F-$ $\left.\varepsilon q, K-t K_{\infty} \backslash\{0\}\right) \subset \overline{\mathbb{B}}(0,1 / \varepsilon)$. By the first part (1), there exists $m>r$, and for any $k \geq m$ and $\varepsilon \in(0,1 / k)$, we have $S\left(F-\varepsilon q, K-t K_{\infty} \backslash\{0\}\right) \neq \emptyset$. Suppose that there exists $q \in$ $\operatorname{int}($ barr $K)$, and for any $k \geq m$, there exists $\varepsilon_{k} \in(0,1 / k)$ such that $x_{k} \in S\left(F-\varepsilon_{k} q, K-t K_{\infty} \backslash\{0\}\right)$; however, $x_{k} \notin \overline{\mathbb{B}}\left(0,1 / \varepsilon_{k}\right)$.

Since $x_{k} \in I$ and $\left\|x_{k}\right\|>1 / \varepsilon_{k}>k \geq m>r$, applying the same method of proof as (1), there exists $\widetilde{x_{k}} \in K$ and $\left\|\widetilde{x_{k}}\right\|>r$. It follows from coercivity condition (30) that there exists $y_{k} \in K_{r}$ such that there exists $y_{k}^{*} \in F\left(y_{k}\right) \cap \operatorname{barr}(K)$, $\left\langle y_{k}^{*}, y_{k}-\widetilde{x_{k}}\right\rangle<0$. Similarly, we can get

$$
\sup _{\xi_{k} \in F\left(x_{k}\right)}\left\langle\xi_{k}, y_{k}-x_{k}\right\rangle \leq 0 .
$$

Moreover, $y_{k} \in K$ implies that $y_{k} \in I$, because $K=I+$ $t K_{\infty}=I+t I_{\infty} \subset I$. By $x_{k} \in S\left(F-\varepsilon_{k} q, K-t K_{\infty} \backslash\{0\}\right)$, we have

$$
\begin{aligned}
0 & \leq \sup _{\xi_{k}^{*} \in F\left(x_{k}\right)}\left\langle\xi_{k}^{*}-\varepsilon_{k} q, y_{k}-x_{k}\right\rangle \\
& =\sup _{\xi_{k}^{*} \in F\left(x_{k}\right)}\left\langle\xi_{k}^{*}, y_{k}-x_{k}\right\rangle+\varepsilon_{k}\left(\left\langle q, x_{k}\right\rangle-\left\langle q, y_{k}\right\rangle\right) \\
& \leq \varepsilon_{k}\left(\left\langle q, x_{k}\right\rangle-\left\langle q, y_{k}\right\rangle\right) .
\end{aligned}
$$

Similarly, we can assume that $x_{k} /\left\|x_{k}\right\| \rightarrow e$. Thus, $\left\langle q, x_{k} /\left\|x_{k}\right\|\right\rangle \rightarrow\langle q, e\rangle<0$. By sign-preserving theorem of limit, there exists $K>0$, when $k>K,\left\langle q, x_{k} /\left\|x_{k}\right\|\right\rangle<0$. Since $\left\|x_{k}\right\|>1 / \varepsilon_{k},\left\|x_{k}\right\| \varepsilon_{k}>1$. When $k>K$,

$$
\varepsilon_{k}\left\langle q, x_{k}\right\rangle=\left\|x_{k}\right\| \varepsilon_{k}\left\langle q, \frac{x_{k}}{\left\|x_{k}\right\|}\right\rangle<0 .
$$

Similarly, $\left\{y_{k}\right\} \subset K_{r}$ implies that $\left\{y_{k}\right\}$ is bounded, $\varepsilon_{k}\left\langle q, y_{k}\right\rangle \rightarrow$ 0 . For large enough $k, \varepsilon_{k}\left(\left\langle q, x_{k}\right\rangle-\left\langle q, y_{k}\right\rangle\right)<0$. It is a contradiction with (40). This completes the proof.
Remark 10. Li and He [6] gave a similar result of $\operatorname{GVIP}(F, K)$ where only the mapping was directional perturbed in $\mathbb{R}^{n}$ (Theorem 3.2 of [6]). Theorem 9 generalizes the result of [6] from $\mathbb{R}^{n}$ to a reflexive Banach space, gives a stronger conclusion, and discusses the case that the mapping is perturbed along a direction and the constraint set is perturbed along another direction simultaneously. Theorem 9 also improves Theorem 3.1 of [30] to the case that the set is also directional perturbed simultaneously.

\section{Conclusions}

The aim of this paper is to improve the solvability results for perturbed generalized variational inequalities to the case that the perturbations act both on the mapping $F$ and on the constraint set $K$. It is important to observe that the second solvability result for perturbed generalized variational inequality when the perturbation terms $q$ and $K_{\infty}$ are along some certain directions simultaneously is new and interesting. Further research works should be carried out to develop the similar solvability results for some other optimization problems with the perturbation terms perturbed along some certain directions.

\section{Data Availability}

No data were used to support this study.

\section{Conflicts of Interest}

The authors declare that there are no conflicts of interest regarding the publication of this paper.

\section{Acknowledgments}

The work was supported by the National Natural Science Foundation of China (Grant 11701480), Fundamental Research Funds for the Central Universities, Southwest University for Nationalities (Grant 2018HQZZ23), Key Projects of the Education Department of Sichuan Province (Grant 18ZA0511), Innovation Team Funds of Southwest University for Nationalities (Grant 14CXTD03), Innovative Research Team of the Education Department of Sichuan Province (Grant 15TD0050), and Sichuan Youth Science and Technology Innovation Research Team (Grant 2017TD0028).

\section{References}

[1] A. Daniilidis and N. Hadjisavvas, "Coercivity conditions and variational inequalities," Mathematical Programming, vol. 86, no. 2, Ser. A, pp. 433-438, 1999.

[2] J.-P. Crouzeix, "Pseudomonotone variational inequality problems: existence of solutions," Mathematical Programming, vol. 78, no. 3, Ser. A, pp. 305-314, 1997.

[3] J. Fan and R. Zhong, "Stability analysis for variational inequality in reflexive Banach spaces," Nonlinear Analysis. Theory, Methods \& Applications. An International Multidisciplinary Journal, vol. 69, no. 8, pp. 2566-2574, 2008.

[4] Y. He, "Stable pseudomonotone variational inequality in reflexive Banach spaces," Journal of Mathematical Analysis and Applications, vol. 330, no. 1, pp. 352-363, 2007. 
[5] Y. He, "The Tikhonov regularization method for set-valued variational inequalities," Abstract and Applied Analysis, Article ID 172061, Art. ID 172061, 10 pages, 2012.

[6] F. Li and Y. He, "Solvability of a perturbed variational inequality," Pacific Journal of Optimization. An International Journal, vol. 10, no. 1, pp. 105-111, 2014.

[7] D. Aussel and N. Hadjisavvas, "On quasimonotone variational inequalities," Journal of Optimization Theory and Applications, vol. 121, no. 2, pp. 445-450, 2004.

[8] M. Bianchi, N. Hadjisavvas, and S. Schaible, "Exceptional families of elements for variational inequalities in Banach spaces," Journal of Optimization Theory and Applications, vol. 129, no. 1, pp. 23-31, 2006.

[9] J. Han, Z. Huang, and S. Fang, "Solvability of variational inequality problems," Journal of Optimization Theory and Applications, vol. 122, no. 3, pp. 501-520, 2004.

[10] I. V. Konnov, "On the convergence of a regularization method for variational inequalities," Computational Mathematics and Mathematical Physics, vol. 46, no. 4, pp. 541-547, 2006.

[11] F. Facchinei and J.-S. Pang, Finite-dimensional variational inequalities and complementarity problems. Vol. I, Springer Series in Operations Research, Springer-Verlag, NY, USA, 2003.

[12] M. Sofonea and Y. Xiao, "Fully history-dependent quasivariational inequalities in contact mechanics," Applicable Analysis: An International Journal, vol. 95, no. 11, pp. 2464-2484, 2016.

[13] Y. Wang, Y. Xiao, X. Wang, and Y. J. Cho, "Equivalence of well-posedness between systems of hemivariational inequalities and inclusion problems," Journal of Nonlinear Sciences and Applications. JNSA, vol. 9, no. 3, pp. 1178-1192, 2016.

[14] W. Li, Y. Xiao, N. Huang, and Y. J. Cho, "A class of differential inverse quasi-variational inequalities in finite dimensional spaces," Journal of Nonlinear Sciences and Applications. JNSA, vol. 10, no. 8, pp. 4532-4543, 2017.

[15] X. Luo, "Quasi-strict feasibility of generalized mixed variational inequalities in reflexive Banach spaces," Journal of Optimization Theory and Applications, pp. 1-16, 2018.

[16] H. Yang, X. Luo, and L. Chen, "Solving adaptive image restoration problems via a modified projection algorithm," Mathematical Problems in Engineering, Art. ID 6132356, 11 pages, 2016.

[17] X. Luo, "Tikhonov regularization methods for inverse variational inequalities," Optimization Letters, vol. 8, no. 3, pp. 877887,2014

[18] X. Luo and J. Yang, "Regularization and iterative methods for monotone inverse variational inequalities," Optimization Letters, vol. 8, no. 4, pp. 1261-1272, 2014.

[19] J. Liu, "Sensitivity analysis in nonlinear programs and variational inequalities via continuous selections," SIAM Journal on Control and Optimization, vol. 33, no. 4, pp. 1040-1060, 1995.

[20] C. D. Ha, "Application of degree theory in stability of the complementarity problem," Mathematics of Operations Research, vol. 12, no. 2, pp. 368-376, 1987.

[21] S. Dafermos, "Sensitivity analysis in variational inequalities," Mathematics of Operations Research, vol. 13, no. 3, pp. 421-434, 1988.

[22] J. Kyparisis, "Parametric variational inequalities with multivalued solution sets," Mathematics of Operations Research, vol. 17, no. 2, pp. 341-364, 1992.

[23] Y. Qiu and T. L. Magnanti, "Sensitivity analysis for variational inequalities," Mathematics of Operations Research, vol. 17, no. 1, pp. 61-76, 1992.
[24] R. L. Tobin, "Sensitivity analysis for variational inequalities," Journal of Optimization Theory and Applications, vol. 48, no. 1, pp. 191-209, 1986.

[25] M. S. Gowda and J.-S. Pang, "Stability analysis of variational inequalities and nonlinear complementarity problems, via the mixed linear complementarity problem and degree theory," Mathematics of Operations Research, vol. 19, no. 4, pp. 831-879, 1994.

[26] X. Wang, W. Li, X. Li, and N. Huang, "Stability for differential mixed variational inequalities," Optimization Letters, vol. 8, no. 6, pp. 1873-1887, 2014.

[27] R. Hu and Y. Fang, "Levitin-Polyak well-posedness by perturbations for the split inverse variational inequality problem," Journal of Fixed Point Theory and Applications, vol. 18, no. 4, pp. 785-800, 2016.

[28] L. McLinden, "Stable monotone variational inequalities," Mathematical Programming, vol. 48, no. 2, (Ser. B), pp. 303-338, 1990.

[29] R. Zhong and N. Huang, "Stability analysis for Minty mixed variational inequality in reflexive Banach spaces," Journal of Optimization Theory and Applications, vol. 147, no. 3, pp. 454$472,2010$.

[30] Z. Li and S. Sun, "Solvability of a perturbed variational inequality," Acta Mathematica Scientia. Series A. Shuxue Wuli Xuebao. Chinese Edition, vol. 36, no. 3, pp. 473-480, 2016.

[31] J.-P. Aubin, Optima and equilibria, vol. 140 of Graduate Texts in Mathematics, Springer-Verlag, Berlin, 1993.

[32] K. Fan, "A generalization of Tychonoff's fixed point theorem," Mathematische Annalen, vol. 142, pp. 305-310, 1961. 


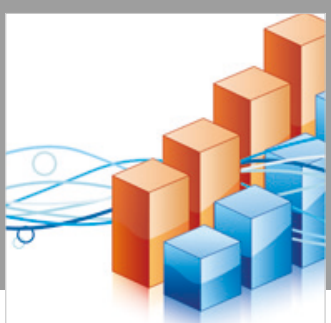

Advances in

Operations Research

\section{-n-m}
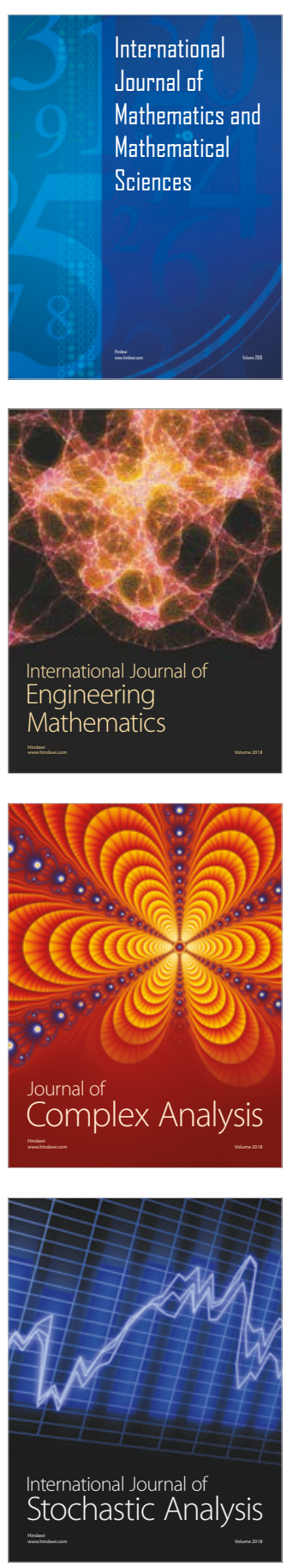
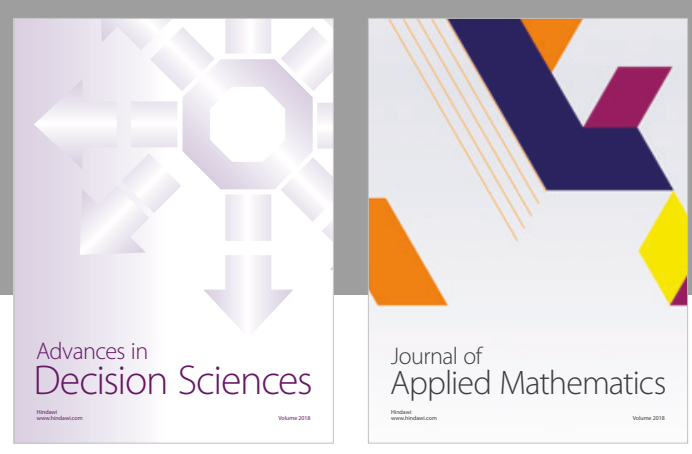

Journal of

Applied Mathematics
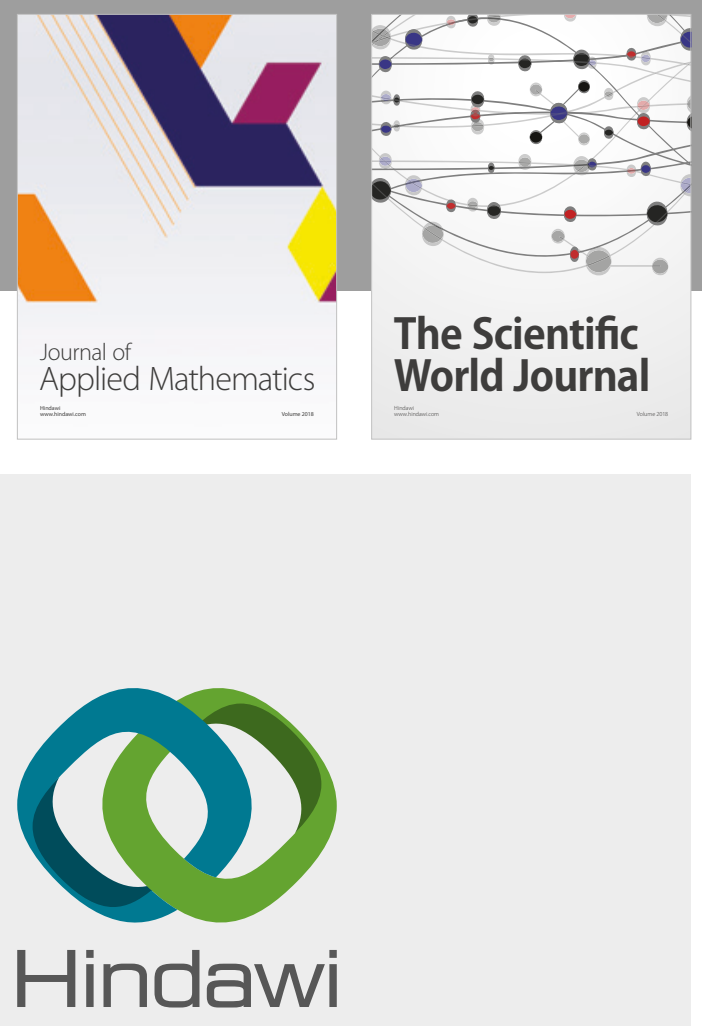

Submit your manuscripts at

www.hindawi.com

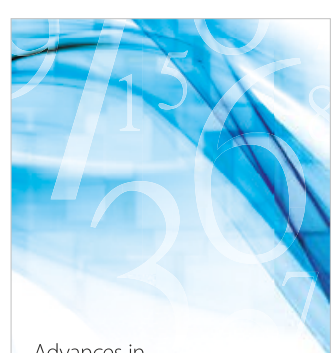

Advances in
Numerical Analysis
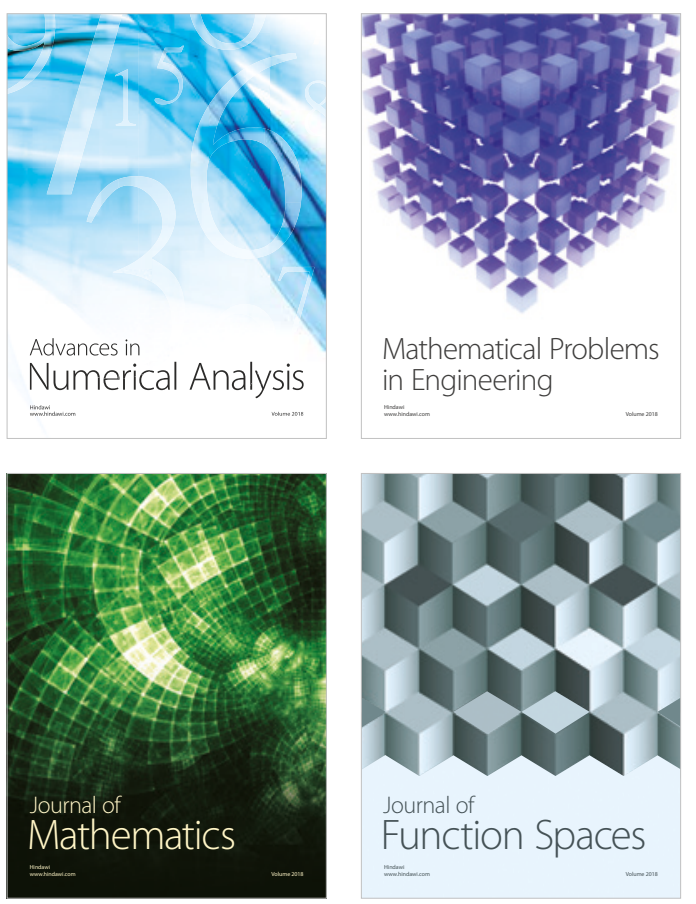

Mathematical Problems in Engineering

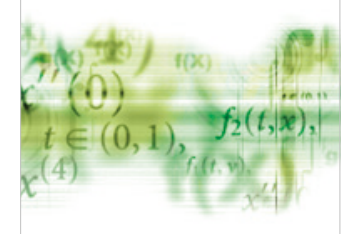

International Journal of

Differential Equations

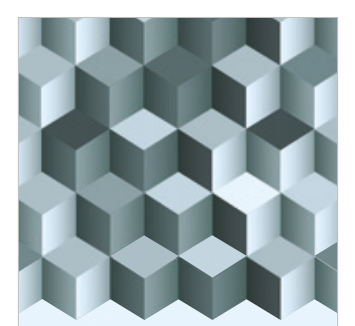

Journal of

Function Spaces

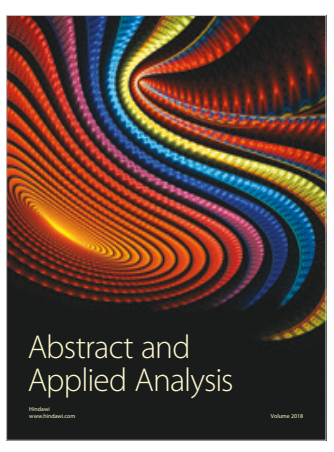

The Scientific

World Journal

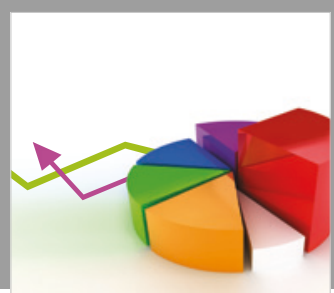

Journal of

Probability and Statistics
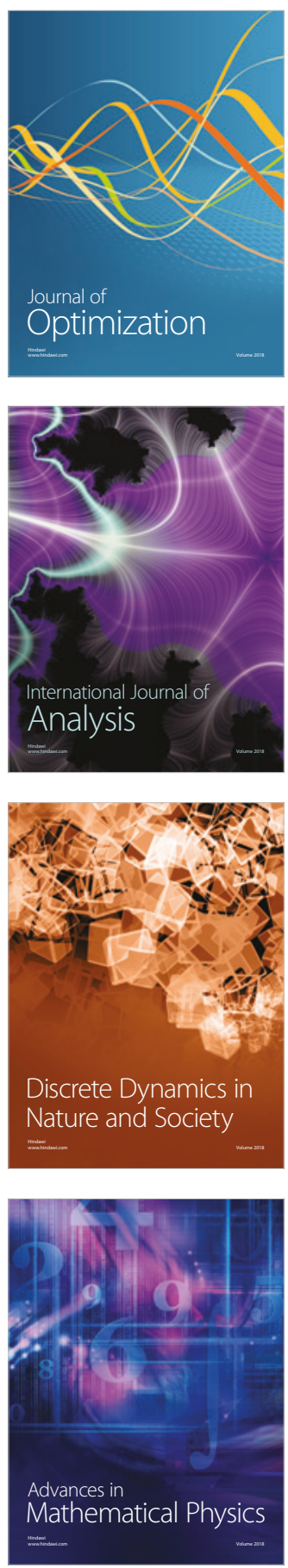